http://jmscr.igmpublication.org/home/ ISSN (e)-2347-176x ISSN (p) 2455-0450 crossref DOI: https://dx.doi.org/10.18535/jmscr/v8i6.55

\title{
Neutrophil to Lymphocyte Ratio as a Marker of Acute Exacerbation and Disease Severity in Chronic Obstructive Pulmonary Disease at Tertiary Care Hospital
}

\author{
Authors \\ Dr Sonam Gupta ${ }^{1}$, Dr Yadvendra Gupta ${ }^{2^{*}}$ \\ ${ }^{1}$ Senior Resident in Internal Medicine, SMS Medical College and Hospital, Jaipur \\ ${ }^{2}$ Senior Resident, Department of Medicine, SMS Medical College and Hospital, Jaipur \\ *Corresponding Author \\ Dr Yadvendra Gupta
}

\begin{abstract}
Background: Our hypothesis is that NLR could be a useful important inflammatory marker that detects the inflammatory status during acute exacerbations of COPD (AECOPD) and could identify early acute exacerbations for early management.

Methods: The cross-sectional study includes patients with Chronic Obstructive Pulmonary Disease irrespective of the severity and duration of disease. Study cases are personally interviewed to get relevant details after getting informed signed consent. Based upon inclusion and exclusion criteria a minimum of 100 cases are selected. An exacerbation of COPD is defined as an onset or worsening of more than two respiratory symptoms (ie, dyspnea, cough or wheeze, sputum amount or purulence) for two or more consecutive days.
\end{abstract}

Results: $46.00 \%$ patients duration of disease was more than 10 year and NLR was $4.45 \pm 1.28$. The severity wise difference in NLR was statistically significant.

Conclusion: NLR can be used as a new inflammatory marker for assessment of inflammation in COPD patients. It is a good predictor for exacerbation of COPD. It is a quick, cheap, easily measurable with no need for a special request.

Keywords: NLR, COPD, Stable.

\section{Introduction}

Chronic obstructive pulmonary disease (COPD) a preventable and treatable disease which consists of constant expiratory limitation of the flow of air which is not fully reversible. A progressive disease, which comprises of enhanced and chronic inflammatory response to gases and noxious materials of the mucosa of respiratory tract.

Detection of COPD is thought in a patient coming with symptoms of cough, excessive production of sputum, or dysponea, and or exposure to factors which causing the disease will be present.

GOLD defines COPD - "Chronic Obstructive Pulmonary Disease (COPD) is a common, preventable and treatable disease that is characterized by persistent respiratory symptoms and airflow limitation that is due to airway and/or alveolar abnormalities usually caused by significant exposure to gases or noxious particles". ${ }^{1}$

One of the characteristic features of COPD is 
acute exacerbations, which usually are associated with increased inflammation due to infections (bacterial, viral and combined viral/ bacteria) and or environmental factors. There is a positive relationship between exacerbations of COPD and hospitalization, decline in quality of life and mortality rate. Early detection of acute exacerbation of COPD is essential to avoid these major complications. ${ }^{2}$ Inflammation in COPD may be contributed to many cell types such as the macrophages, the neutrophils and the lymphocytes 3,4

Fig 1 Classification of severity of air flow limitation in COPD as per GOLD criteria

\begin{tabular}{|l|l|l|}
\multicolumn{3}{|c}{ Classification of COPD by impairment of lung function } \\
\hline Stage & Severity & Spirometry (postbronchodilator) \\
\hline GOLD 1 & Mild & $\begin{array}{l}\mathrm{FEV}_{1} 280 \% \text { predicted } \\
\mathrm{FEV}_{1} / \mathrm{FVC}<0.7\end{array}$ \\
\hline GOLD 2 & Moderate & $\begin{array}{l}50 \% \leq \mathrm{FEV}_{1}<80 \% \text { predicted } \\
\mathrm{FEV}_{1} / \mathrm{FVC}<0.7\end{array}$ \\
\hline GOLD 3 & Severe & $\begin{array}{l}30 \% \leq \mathrm{FEV}_{1}<50 \% \text { predicted } \\
\mathrm{FEV}_{1} / \mathrm{FVC}<0.7\end{array}$ \\
\hline GOLD 4 & Very severe & $\begin{array}{l}\mathrm{FEV} V_{1}<30 \% \text { predicted } \\
\mathrm{FEV} / \mathrm{FVC}<0.7\end{array}$ \\
\hline
\end{tabular}

Neutrophils play an important role in inflammatory conditions more than macrophages. Neutrophils are an important source of proteases, especially reactive oxygen species and neutrophilelastase. They are the hallmark of acute inflammation. ${ }^{5}$

Unlike other inflammatory biomarkers e.g., ESR and CRP, the Neutrophil-lymphocyte ratio (NLR) is derived from routine complete blood count (CBC) tests. It does not need a special request. It is also a rapid, easy method, and cost-effective. Many studies have reported an increase of NLR during the inflammatory conditions in different diseases such as pancreatitis, inflammatory bowel diseases, and acute coronary syndrome ${ }^{6-10}$

To our knowledge few number of studies have been published about the relationship between neutrophil lymphocyte ratio and respiratory diseases.
Our hypothesis is that NLR could be a useful important inflammatory marker that detects the inflammatory status during acute exacerbations of COPD (AECOPD) and could identify early acute exacerbations for early management.

The aim of this study is to evaluate the potential for NLR to be used as a biomarker of COPD exacerbation.

\section{Materials and Methods}

Design of Study: Cross Sectional Study

Sample Size: 100 patients

The study includes patients with Chronic Obstructive Pulmonary Disease irrespective of the severity and duration of disease. Study cases are personally interviewed to get relevant details after getting informed signed consent. Based upon inclusion and exclusion criteria a minimum of 100 cases are selected. An exacerbation of COPD is defined as an onset or worsening of more than two respiratory symptoms (ie, dyspnea, cough or wheeze, sputum amount or purulence) for two or more consecutive days.

\section{Inclusion Criteria}

Stable diagnosed COPD patients of age 40 years or older who were current or ex smokers based on clinical history and examination

\section{Exclusion Criteria}

- age < 40years

- Patients with and diagnosed as Bronchial Asthma, Bronchiectasis or Bullous lung disorders.

- Patients with active pulmonary tuberculosis.

- Patients with malignancy.

- Patients with hepatic disease, renal disease, myocardial infaction.

- Patients with any other acute or chronic infections.

- Patients with pneumonia.

- Patients with dementia.

- Patients with Diabetes Mellitus.

- Patients receiving systemic corticosteroids, antibiotics.

- Patients receiving immunosuppressive treatment. 


\section{Methods of Study}

Data is collected using pretest proformas according to the objectives of the study. After getting informed signed consent detailed history and examination was done in 100 patients included in the study.

Those patients who satisfied all the inclusion and exclusion criteria were selected for the study

\section{Investigations}

\section{1) Routine Complete Blood count}

TC, DC (including neutrophil and lymphocyte counts), Hemoglobin percentage.

2) Blood Biochemistry

Random blood sugar Blood urea

Serum creatinine

Serum electrolytes: Sodium, Potassium

3) ECG

4) Imaging Studies

CT Chest

Chest $\mathrm{X}$ ray $\mathrm{PA}$ view

5) Pulmonary Function Test

6) Sputum AFB, sputum culture and sensitivity (if needed)

\section{Statistical Methods}

All the data were entered in a data collection sheet in an Excel format and analysed using SPSS Software. Numerical values were reported using mean and standard deviation or median. Categorical values are reported using number and percentages. Probability value (p) value less than 0.05 was considered a statistically significant.

\section{Results}

Table No: 1: Age Distribution Age in Years

\begin{tabular}{|l|c|c|}
\hline Age (Yrs) & No of patients & NLR \\
\hline$<50$ & 9 & $4.8 \pm 1.34$ \\
\hline $51-60$ & 31 & $3.87 \pm 1.8$ \\
\hline $61-70$ & 48 & $3.62 \pm 1.71$ \\
\hline$>70$ & 12 & $4.2 \pm 1.4$ \\
\hline
\end{tabular}
p-value $=0.123$

$48.00 \%$ patients were from $61-70$ years age groups and NLR was $3.87 \pm 1.8$,
Table No: 2. Sex Distribution

\begin{tabular}{|l|c|c|}
\hline SEX & No of patients & NLR \\
\hline Male & 82 & $3.83 \pm 1.5$ \\
\hline Female & 18 & $3.89 \pm 1.46$ \\
\hline
\end{tabular}

p-value $=0.242$

$88.00 \%$ patients were male and NLR was $3.83 \pm 1.5$.

Table No: 3. Duration Wise Distribution

\begin{tabular}{|l|c|c|}
\hline DURATION (Yrs) & No of patients & NLR \\
\hline$<5$ & 30 & $2.71 \pm 1.47$ \\
\hline $5-10$ & 24 & $3.99 \pm 1.48$ \\
\hline$>10$ & 46 & $4.45 \pm 1.28$ \\
\hline \multicolumn{3}{|l}{ p-value $=0.04$}
\end{tabular}

$46.00 \%$ patients duration of disease was more than 10 year and NLR was $4.45 \pm 1.28$.

Table No: 4. Severity Wise Distribution.

\begin{tabular}{|l|c|c|}
\hline SEVERITY & No of patients & NLR \\
\hline Exacerbation & 38 & $4.56 \pm 1.81$ \\
\hline Stable & 62 & $2.35 \pm 1.16$ \\
\hline
\end{tabular}

The severity wise difference in NLR was statistically significant.

\section{Discussion}

The study is a cross sectional study pattern where neutrophil lymphocyte ratio was obtained for the COPD patients included in the study, and their disease severity and long term outcome is predicted.

The patients who underwent the study were clinically examined along with detailed history was taken and complete blood count and spirometry was done along with the routine investigations.

It is well known that the leukocyte count and its subtypes are inflammatory markers. ${ }^{11,12}$ On exposure to a physiological stress the leukocyte count changes in the circulation. The number of neutrophils increase, while, the number of lymphocytes decrease. Some authors used the ratio of these subgroups to each other as a marker of inflammation ${ }^{13}$ AE-COPD is accompanied with deterioration in lung function. Acute exacerbation of COPD increases the hospitalization rate, decreases the lung function, and deteriorates the patient's quality of life. Severe attacks of 
exacerbated COPD lead to increased mortality raterker. Presence of a marker for early detection of the of $\mathrm{AE}$ is essential to avoid these major complications. ${ }^{13}$

NLR is a cheap inflammatory marker that can be obtained from the routine laboratory investigations. It has a predictive and prognostic values in systemic inflammatory diseases such as inflammatory bowel disease, kidney disease, cardiovascular disease, and familial Mediterranean fever. ${ }^{14}$

We aimed in this study to evaluate the potential for NLR to be used as a biomarker of COPD exacerbation. In the current study, we found that the blood leukocytic count, ESR and CRP was higher in stable COPD patients than in healthy subjects. These results indicate that there is a low grade persistent systemic inflammation even in stable COPD patients. This was described in previous studies ${ }^{15}$.

\section{Conclusion}

NLR can be used as a new inflammatory marker for assessment of inflammation in COPD patients. It is a good predictor for exacerbation of COPD. It is a quick, cheap, easily measurable with no need for a special request.

\section{References}

1. Global Initiative for Chronic Obstructive Lung Disease (GOLD): Global Strategy for the Diagnosis, Management and prevention of Chronic Obstructive Pulmonary Disease. (2017 REPORT). Available from: http://www.goldcopd.org

2. Mathers CD, Loncar D. Projections of global mortality and burden of disease from 2002 to 2030. PLoS medicine. 2006; 3(11):e442.

3. Yin P, Wang H, Vos T, Li Y, Liu S, Liu $\mathrm{Y}$, et al. A Subnational Analysis of Mortality and Prevalence of COPD in China From 1990 to 2013: Findings From the Global Burden of Disease Study 2013. Chest. 2016; 150(6):1269-80.
4. Perera PN, Armstrong EP, Sherrill DL, Skrepnek GH. Acute exacerbations of COPD in the United States: inpatient burden and predictors of costs and mortality. Copd. 2012; 9(2):131-41.

5. Celli BR, MacNee W. Standards for the diagnosis and treatment of patients with COPD: a summary of the ATS/ERS position paper. The European respiratory journal. 2004; 23(6):932-46.

6. Zahorec R. Ratio of neutrophil to lymphocyte counts-rapid and simple parameter of systemic inflammation and stress in critically ill. Bratislavskelekarskelisty. 2001; 102(1):514.

7. Liu CL, Lee JJ, Liu TP, Chang YC, Hsu YC, Cheng SP. Blood neutrophil-tolymphocyte ratio correlates with tumor size in patients with differentiated thyroid cancer. Journal of surgical oncology. 2013; 107 (5):493-7

8. Templeton AJ, McNamara MG, Seruga B, Vera-Badillo FE, Aneja P, Ocana A, et al. Prognostic role of neutrophil-tolymphocyte ratio in solid tumors: a systematic review and meta-analysis. Journal of the National Cancer Institute. 2014; 106(6):dju124.

9. Bhat T, Teli S, Rijal J, Bhat H, Raza M, Khoueiry G, et al. Neutrophil to lymphocyte ratio and cardiovascular diseases: a review. Expert review of cardiovascular therapy. 2013; 11(1):55-9.

10. de Jager CP, van Wijk PT, Mathoera RB, de Jongh-Leuvenink J, van der Poll T, Wever PC. Lymphocytopenia and neutrophil-lymphocyte count ratio predict bacteremia better than conventional infection markers in an emergency care unit. Critical care (London, England). 2010; 14(5):R192. Epub 2010/11/ 03. https://doi.org/10.1186/cc9309 PMID: 21034463.

11. Zhou X, Li Q, Zhou X. Exacerbation of 
chronic obstructive pulmonary disease.

Cell BiochemBiophys. 2015;73(2):349355.

12. Groenewegen KH, Postma DS, Hop WC, Wielders PL, Schlösser NJ, Wouters EF; COSMIC Study Group. Increased systemic in am- mation is a risk factor for COPD exacerbations. Chest. 2008;133(2): 350357.

13. Faria SS, Fernandes PC Jr, Silva MJ, et al. The neutrophil-tolymphocyte ratio: a narrative review. Ecancermedicalscience. 2016; 10:702.

14. Yang $\mathrm{T}$, Wan $\mathrm{C}$, Wang $\mathrm{H}$, et al. The prognostic and risk-strati ed value of neutrophil-lymphocyte count ratio in Chinese patients with community-acquired pneumonia. Eur J In amm. 2017;15(1):2227. 50

15. de Jager CP, Wever PC, Gemen EF, et al. The neutrophillymphocyte count ratio in patients with community-acquired pneumonia. PLoS One. 2012;7 (10):e46561. 